\title{
ARTÍCULOS
}

CRITICA, Revista Hispanoamericanc de Filosofia

Vol. XXII, No. 65 (agosto 1990): 3-21

\section{LA OBJETIVIDAD DEL AZAR EN UN MUNDO DETERMINISTA}

SERGIO MARTÍNEZ

Instituto de Investigaciones Filosóficas Universidad Nacional Autónoma de México

\section{Introducción}

Hay una familia problemática de conceptos en los fundamentos de la física de profunda raigambre filosófica. A través de la historia de la filosofía han sido confundidos, falsamente identificados, incorrectamente diferenciados, y las más de las veces simplemente ignorados. Miembros notables de esta familia son los conceptos de posibilidad física, necesidad causal, determinismo y probabilidad irreductible. En este trabajo examinaremos importantes conexiones entre miembros de esta familia de conceptos centrándonos en el estudio de un miembro de esta familia, no tan famoso como sus congéneres, pero, creemos nosotros, si tan importante: el concepto de azar físico (objetivo).

Hay tres áreas fundamentales en los fundamentos de la ciencia (con fronteras y traslapes fluctuantes) en las que una clarificación del concepto de azar se perfila como una empresa filosófica importante. Parece ser que la formulación de un concepto de azar objetivo es crucial para la formulación de un esquema inductivo de explicación, ya que el uso de un concepto epistémico de azar trivializa el modelo estadístico de ex- 
plicación de Hempel (el modelo I-S). ${ }^{1}$ Hay muchas otras propuestas para un análisis del pensamiento inductivo que se basan directa o indirectamente en teorías del azar. Por ejemplo, Solomonoff (1964) ha propuesto una teoría de inferencia inductiva, basada en una formalización del concepto de sucesión azarosa que es esencialmente el mismo formalizado por Kolmogorov (y que examiné en Martínez 1990a). ${ }^{2}$ Por otro lado, una tradición importante en el estudio de los fundamentos matemáticos y filosóficos de la teoría de la probabilidad sostiene que una clarificación del concepto de azar podría permitir una formulación adecuada de una interpretación de la probabilidad por medio de frecuencias relativas. La idea básica aquí es que el problema de la interpretación de las probabilidades, sobre todo la clarificación de la relación del cálculo de probabilidades con la estructura causal del mundo, requiere la delimitación del dominio de aplicación de la teoría de la probabilidad. Otra área de interés, y en la que este trabajo se enmarca, radica en la posibilidad de formular conexiones esclarecedoras entre el concepto de azar y otros miembros de nuestro grupo problemático de conceptos, a través de un análisis de procesos azarosos en teorías específicas.

Está bastante extendida la idea de que el azar es un concepto meramente matemático y de que es solo el producto de una confusión de categorías el pensar en algo como un 'azar físico'. Esta idea proviene del predominio de las tendencias reduccionistas en la filosofía de la ciencia asociadas al programa empirista. Este trabajo es un esfuerzo por examinar el concepto de azar desde un punto de vista diferente, no reduccionista. Nos interesa en particular formular una serie de distinciones y relaciones entre nuestros concepios problemáticos (basadas en

1 Un examen de esta dificultad para un modelo estadístico de explicación se puede encontrar en Salmon (1984).

2 Otros aspectos de la relación entre la formulación de un concepto de azar y el problema de la caracterización del conocimiento inductivo se examinan en Salmon (1984), Skirms (1984). 
el examen de teorías específicas), que nos lleven a una clarificación del concepto de probabilidad objetiva, y sobre todo, de cierto tipo de probabilidades irreductibles (propensiones).

Es indudable que el desarrollo de la ciencia moderna a partir de Newton se ha esforzado en mostrar la dispensabilidad de conceptos modales en la ciencia. Así, el newtonianismo, sobre todo a partir de la formulación de Lagrange, se formula como una teoría deductiva de la mecánica que no requiere de ninguna noción física irreductible de causa para explicar el movimiento mecánico de los cuerpos. En la teoría de la relatividad de Einstein se reifican la trayectorias luminosas posibles sobre la base de las cuales se describe la geometría del espacio tiempo. En la mecánica estadística el espacio de fase reifica las posibles trayectorias de un sistema, lo que permite un análisis puramente extensional (conjuntista) de la dinámica de sistemas estadísticos.

Así, el empirista, al seguir supuestamente las huellas de la ciencia, parte de la presuposición de que las probabilidades que aparecen en nuestras explicaciones y teorías cientificas deben de ser en último término reductibles a sistematizaciones de hechos ocurrentes. Y que, por lo tanto, la objetividad de las probabilidades debe de poder asirse por medio de una definición que sirva de base a todas las aplicaciones de la teoría matemática de la probabilidad. Esta es una presuposición cuestionable. Es posible que la objetividad de las probabilidades no pueda asirse a través de una interpretación válida en toda la extensión de la ciencia; es posible que el sentido en el que las probabilidades son propiedades (objetivas) de sistemas físicos sólo pueda asirse de manera precisa por medio de teorías o campos de aplicación específicos. Las dificultades ya bastante conocidas con las diferentes interpretaciones de la probabilidad sugieren que ésta es una posibilidad digna de explorarse seriamente. Además, tenemos que reconocer que en una serie de teorías modernas en la física y en la biología, y en general en la ciencia moderna, se maneja una noción de 
azar que se expresa por medio de probabilidades o de leyes estadísticas que se consideran "irreductibles". El sentido en el que estas probabilidades pueden considerarse "irreductibles" no es claro pero, como veremos, esta falta de claridad surge en buena medida de una falta de distinciones relevantes con respecto a los conceptos de azar y determinismo.

2. El concepto de probabilidad irreductible y la tesis del determinismo

En la filosofía de la ciencia está bastante extendida la idea de que la cuestión de si el mundo es determinista o indeterminista es equivalente a la pregunta de si existen probabilidades irreductibles. Autores como Popper (1962) y Stegmüller (1973) y Giere (1973) consideran que la mecánica clásica no admite probabilidades irreductibles, únicamente probabilidades interpretadas subjetivamente como grados de creencia. Paralela a esta idea está también la creencia de que el surgimiento de la mecánica cuántica lleva consigo por primera vez la posibilidad de fundamentar un concepto de probabilidad irreductible. Hay una pizca de verdad en esta leyenda filosófica pero, como veremos, no mucho más que una pizca.

En esta sección estableceremos una distinción importante entre la presencia de probabilidades 'irreductibles' y la tesis del indeterminismo (causal) que nos servirá de punto de partida para nuestro estudio.

La doctrina clásica del determinismo fue formulada por Laplace en (1814) en un párrafo famoso:

Una inteligencia que por un instante pudiera comprender todas las fuerzas que animan a la naturaleza y la situación respectiva de los seres que la componen -una inteligencia suficientemente vasta para someter todos estos datos a análisis - abarcaría en la misma fórmula los movimientos de los cuerpos más grandes del universo y los de los átomos más ligeros, para él nada sería incierto, y el futuro, así como el pasado, estarían presentes a sus ojos. (p. 4) 
Debemos empezar haciendo explícitas una serie de distinciones importantes para nuestra tarea. Debemos distinguir en particular la tesis metafísica del determinismo de una serie de importantes implicaciones de la formulación de Laplace que tienen más bien un caracter epistemológico o metodológico. La confusión entre estas ideas sutilmente entrelazadas en la doctrina laplaciana ha llevado al garete a muchos intentos por abordar el tema.

Antes que nada, debemos hacer una distinción explícita entre la noción epistémica de predictibilidad (en principio) y la tesis metafísica del determinismo. Es posible que no podamos predecir el estado futuro de un sistema porque la función que describe el movimiento no es computable, por ejemplo, pero que ese estado futuro esté dado desde siempre y sea único. ${ }^{3} \mathrm{La}$ doctrina laplaciana del determinismo tiene también importantes implicaciones metodológicas profundamente enraizadas en la ciencia moderna, que van más allá de la cuestión de si el mundo físico es o no determinista en un sentido estricto (ver la definición de Montague-Earman, abajo). Una implicación metodológica importante para nosotros es que las atribuciones de probabilidad son justificadas sólo si reflejan la ignorancia de ciertos hechos. Otra implicación metodologica es la tesis de la separabilidad: un sistema o proceso puede caracterizarse cabalmente presuponiendo que es aislable de otros sistemas o procesos en el universo. Estas dos implicaciones metodológicas de la doctrina laplaciana, que son logicamente independientes de la tesis metafísica del determinismo, se combinan para negar la posibilidad de interpretar las probabilidades como medida de propiedades irreductiblemente relacionales y, en general, tienen el efecto de apoyar lo que llamaremos la tesis ontolbgica de la separabilidad: un sistema o proceso puede caracterizarse

3 Aquí no puedo detenerme a clarificar más a fondo esta distinción. Ver Earman (1986), capítulo II, donde se hace ver claramente la distinción entre predictibilidad y determinismo comparando las definiciones de Popper y Russell. 
completamente en términos de las propiedades que un sistema tiene en un momento dado (independientemente de las propiedades de otros sistemas). La noción de completitud en cuestión puede clarificarse como sigue. Un estado consiste en una lista de las propiedades que el sistema tiene en un momento dado. Esta lista es completa cuando permite la deducción de cualquier otra propiedad presente o futura.

La confusión entre conceptos epistémicos (como predictibilidad y confirmación) y principios metodológicos como los arriba mencionados, con la tesis determinista, ha sido uno de los obstáculos más serios para la formulación de esta tesis filosófica. Precisamente esta independencia de nociones epistémicas y principios metodológicos es una de las ventajas de la siguiente definición de determinismo:

Definición (Montague-Earman): Una teoría $T$ es determinista con respecto a un conjunto de magnitudes $M$ si para cada par de mundos físicamente posibles la teoría satisface la condición de que si las magnitudes en cuestión coinciden en un tiempo dado, entonces coinciden para todo tiempo y para toda magnitud en $M$.

Un mundo posible es una colección de sucesos posibles que representan posibles historias alternativas del mundo presente. Un mundo es físicamente posible si satisface todas las leyes de la naturaleza que son el caso en el mundo presente. Earman explora, a partir de esta definición, las condiciones que una teoría debe satisfacer para que podamos decir que el mundo es desterminista con respecto a las variables de una teoría. Nuestro interés presente, sin embargo, no radica en un estudio de la aplicabilidad de esta definición, ni y en el examen de su importancia. Remitimos al lector a Earman (1986) para tal efecto.

Brevemente, sin embargo, queremos hacer ver algunas dificultades de fondo con esta definición. Por un lado es claro que, de alguna forma, la aplicación de esta definición requiere la justificación de la idea de que las magnitudes de una teoría 
no son meras descripciones extensionales de las variables de una teoría. Esto es, tenemos que garantizar o presuponer de alguna forma (sobre la base de una teoría de la causalidad física, por ejemplo), que las magnitudes de una teoría se refieren "rígidamente" (en el sentido de Kripke) a un aspecto del mundo físico. De no ser así es fácil transformar cualquier teoría indeterminista en el sentido de Montague-Earman en una teoría determinista ( $\mathrm{y}$ viceversa). Todo lo que tendría que hacerse es redefinir los mundos físicamente posibles de tal manera que la nueva clasíficación establezca una nueva relación de igualdad de mundos posibles. ${ }^{4}$

No obstante, y esto es lo importante para nosotros, la definición de Montague-Earman nos permite separar explícitamente la cuestión de la existencia de probabilidades irreductibles, de una tesis determinista. Que el determinismo falle no implica la existencia de algún elemento irreductiblemente estocástico en la naturaleza. Tal y como Earman muestra en 1986, la falla del determinismo laplaciano en teorías físicas como la mecánica newtoniana no requiere consideraciones probabilistas. Como veremos en este trabajo el converso de esta tesis también falla. En un sentido que es problemático, pero que trataremos de clarificar abajo, la existencia de probabilidades 'irreductibles' es compatible con un mundo determinista. En la sección siguiente examino una propuesta concreta para la formulación de un concepto de azar objetivo, que sustentaría un tipo de 'probabilidades irreductibles', en una teoría estadística clásica. Como veremos, la dificultad radica en formular una noción de probabilidad irreductible que no es totalmente epistémica.

4 Por ejemplo, si las leyes de la naturaleza son meras descripciones de regularidades extensionales, podemos reagrupar estas descripciones de manera diferente, como en el famoso ejemplo de las esmeraldas verdiazules de Goodman, para adecuarlas de manera ad-hoc a la definición de determinismo en cuestión. 


\section{Azar objetivo en la teoría ergódica}

Examinaremos en esta sección una noción de azar de la cual se sostiene con frecuencia que la la teoría ergódica permite fundamentarla. Se dice que una transformación dinámica determinista $T$ satisface la propiedad de 'mezcla' (mixing) con respecto a una medida $P$, si $\lim P\left(A^{n} T B^{n}\right)=P(A) P(B)$, donde $A$ y $B$ son conjuntos del conjunto de estados de un sistema dinámico. Una trasformación $T$ satisface la condición de mezcla si las correlaciones entre estados en diferentes regiones desaparecen asintóticamente. Mezcla es una de las así llamadas "condiciones de azar". Podemos ilustrar el significado físico de esta condición de azar de la siguiente manera. Dejemos caer una gota de tinta en un vaso de agua. En el momento de caer la tinta está bien localizada. Hay una frontera relativamente clara entre los puntos del espacio dentro del vaso (que podemos identificar con estados del volumen de agua) donde está la tinta, y los puntos donde sólo hay agua. Pero muy pronto, sobre todo si agitamos el vaso, la tinta estará distribuida homogéneamente en todo el volumen de agua. La tinta, podríamos decir, "olvidó" su procedencia de una gota en particular; ya no hay ninguna correlación que nos recuerde que originalmente la tinta estaba concentrada en una región particular del vaso.

Intuitivamente esta condición de "mezcla" es una condición de azar en el sentido siguiente. Marquemos una de las moléculas de la gota de tinta de alguna manera. Si nos preguntamos en que región del volumen del vaso estará nuestra molécula marcada después de un tiempo relativamente largo (digamos un día), la respuesta es que no es posible predecir tal cosa, su posición al día siguiente es impredictible. Todas las regiones del vaso tienen la misma probabilidad de que encontremos en ellas la molécula después de este tiempo relativamente largo.

Ahora bien, ien que sentido de posible no es posible predecir la posición final de la gota? Seguramente que no queremos decir que tal predicción no es lógicamente posible. En tanto que 
aceptamos la posibilidad lógica de aislar la gota y de fijar las condiciones iniciales de la gota, es lógicamente posible predecir la posición final de nuestra molécula marcada. Debemos, pues, entender que la predicción es fisicamente imposible.

La fundamentación que se da usualmente de esta idea de "imposibilidad física", y del azar objetivo asociado con esta fundamentación, es la tesis de la granulación de nuestros instrumentos ("coarse graining"). Según esta tesis, nuestros instrumentos sólo pueden tener una precisión finita que hace imposible algunas preparaciones de estado, como la preparación de estado que requeriría la determinación de las condiciones iniciales de la molécula marcada. Esta determinación requeriría a su vez la determinación de las correlaciones con todas las otras moléculas del volumen de agua con precisión infinita. Sin embargo, puesto que toda medición y preparación requiere una cierta cantidad de energía y procesamiento de información, tal determinación de las condiciones iniciales con precisión infinita requeriría, por lo menos en el caso de sistemas caóticos, ${ }^{5}$ una cantidad infinita de energía. Por lo tanto, si asumimos que la cantidad de energía a nuestra disposición (en nuestra región del universo) es finita, debemos de concluir que es físicamente imposible el determinar la posición final de la molécula de tinta y, por lo tanto, la descripción estadística basada en la suposición de una distribución azarosa de las moléculas es a todo lo que podemos aspirar.

Esta fundamentación de la idea de azar objetivo, que se encuentra implícita en diferentes exposiciones de la teoría ergódica, ${ }^{6}$ tiene que confrontar dificultades serias. Intuitivamente, cualquiera que haya visto armar el cubo de Rubik tendrá una idea del problema. Para alguien que no sabe como ordenarlo los

5 Sistemas caóticos son aquellos para los que la predicción de su movimiento requiere una precisión que aumenta exponencialmente con la separación temporal.

6 Ver Hopf (1934), Krylov (1979), Prigogine (1980), von Plato (1982), (1988). 
diferentes colores del cubo parecen situados totalmente al azar. Para alguien que sabe como ordenarlo, los cubos componentes coloreados están situados en un orden que les permite reordenarlos con relativa rapidez. El desorden (o caos) de la configuración inicial es sólo aparente. Nuestra experiencia cotidiana está llena de ejemplos de este tipo de "azar aparente". La criptografía puede proporcionarnos incontables ejemplos más.

Un ejemplo moderno ilustrativo de cómo puede generarse este tipo de azar aparente en la teoría moderna de transformaciones dinámicas (una subteoría de la mecánica moderna) es la, así llamada, "transformación del panadero". Aquí sólo daremos una descripción intuitiva de la transformación. Una descripción más detallada puede eneontrarse en el Apéndice A en Prigogine (1980).

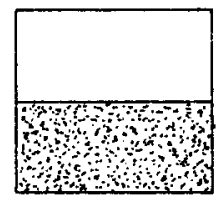

Fig. la

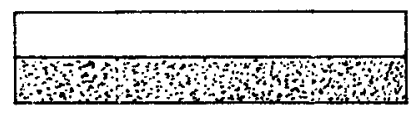

Fig. lb

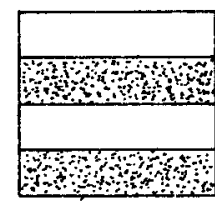

Fig. 1c

Partimos de un cuadrado unitario (figura la) en el cual la mitad inferior tiene alguna propiedad distintiva (color por ejemplo). Como primer paso este cuadrado se aplana (y estira) hasta formar un rectángulo $1 / 2 \times 2$ (figura $1 \mathrm{~b}$ ). Cortemos este rectángulo por la mitad y pogamos una mitad encima de la otra para obtener el cuadrado en la figura lc. A esta transformación del cuadrado la llamaremos transformación $P$. Si continuamos aplicando esta transformación $P$ al cuadrado resultante llegará un momento que no va a importar que tan precisa sean nuestras observaciones (asumiendo que tienen un límite finito de precisión); el cuadrado final nos parecerá totalmente homogéneo. 
Si la propiedad distintiva del rectángulo inferior en el cuadrado inicial era un pigmento de algún color, después de un número suficientemente grande de transformaciones $P$ cualquier muestra que tomemos nos parecerá homogéneamente coloreada.

El resultado de esta transformación es una distribución homogénea aparentemente cática. Para alguien que sabe como fue construida, sin embargo, es fácilmente reversible a la situación original. El caos es solo aparente. Esto es, la distribución azarosa que encontramos en nuestras observaciones satisface todos los criterios matemáticos de azar que queramos imponer, pero físicamente el proceso no está desordenado en el sentido en que existen correlaciones en el sistema que nos permiten reconstruir el estado inicial. Podemos proceder en reversa, paso por paso y reconstituir el cuadrado original. Este tipo de ejemplos de transformaciones generadoras de azar aparente, que pueden ejemplificarse con ejemplos de procesos físicos concretos, ${ }^{7}$ nos confronta con la dificultad siguiente.

En una manera de describir un cierto fenómeno físico, esto es, en un cierto sistema de coordenadas el resultado puede parecer azaroso, mientras que en otro sistema de coordenadas podemos observar un estado perfectamente ordenado. El orden es "subjetivo" en tanto que depende de la selección de un sistema de coordenadas. El problema es la distinción entre azar aparente y azar objetivo. En tanto que una distinción clara, basada en la estructura del mundo físico, no pueda formularse, puede pensarse que un proceso azaroso cualquiera que nos parece objetivo está realmente ordenado para algún observador ideal. ¿Podemos fundamentar la objetividad del azar clásico sin recurrir en última instancia a las limitaciones epistémicas de un

7 Un ejemplo famoso (Hahn 1953) es el de sistemas de espines nucleares en cristales. Los núcleos se preparan con espines paralelos. Conforme pasa el tiempo el sistema de núcleos evoluciona a un sistema que muestra una distribución 'azarosa' de los ejes de los spines. Pero el azar es sólo aparente, se puede revertir el movimiento precesional de los spines hasta que regresan a su estado original con los ejes de los espines paralelos. 
observador ideal? Una manera de intentar esto es recurriendo a una teoría de algoritmos. Caracterizemos una transformación azarosa como aquella que genera estados que no pueden computarse. Más precisamente, podemos partir de la hipótesis de que cualquier estado que puede especificarse por medio de un algoritmo explícito puede también prepararse experimentalmente $\mathrm{y}$, por lo tanto, una transformación generadora de azar objetivo sería aquella que no puede representarse por medio de un algoritmo explícito. ${ }^{8}$ Esta propuesta sin embargo enfrenta la dificuliad de que no es nada claro que es un 'algoritmo explícito'. Cuando nos restringimos a algoritmos familiares y a funciones enteras podemos recurrir a la tesis de Church para clarificar la noción de computabilidad y algoritmo de de manera satisfactoria. Pero cuando estamos hablando de funciones de variable real o compleja tenemos que apelar a una noción de computabilidad generalizada sobre la que no existe, ni parece que pueda existir, un resultado como la tesis de Church que permita clarificar la noción de computabilidad en cuestión.

Enfrentaríamos entonces la necesidad de clarificar la noción de computabilidad (no epistémica) requerida para la fundamentación de la noción objetiva de azar clásico. Esta es indudablemente una tarea que puede llevar a clarificar el contenido físico de la noción matemática de computabilidad, pero que no necesariamente clarificaría importantes aspectos de la conexión entre el azar objetivo y las probabilidades ineductibles que buscamos aquí.

\section{Una interpretación diferente de la irreductibilidad}

El tipo de irreductibilidad en teorías estadísticas clásicas que hemos examinado en la sección anterior se trata de fundamentar en la imposibilidad de observar con la precisión necesaria cierto tipo de sucesos. Se considera que esta irreductibilidad

8 Esta idea ha sido propuesta en muchas variantes, ver por ejemplo Bennett (1985), Peres (1987). 
es objetiva en tanto que depende de la existencia de una cantidad finita de energía en el universo. Esta caracterización de la objetividad del azar incorpora un factor subjetivo importante en tanto que no parece ser posible el formular un criterio claro para separar un azar aparente de un azar objetivo. Esto sucede porque el recurrir a un observador ideal nos remite a una dependencia (intrínseca a la idea de observador) de un sistema de coordenadas. Si tratamos de superar esta dependencia, por medio de una formulación basada en la idea de computación de un algoritmo, enfrentamos el problema de la falta de claridad que existe respecto a las nociones de algoritmo y computabilidad necesarias para llevar a cabo esta tarea.

Sugiero a continuación otra manera de fundamentar la objetividad del azar en teorías estadísticas clásicas. Los intentos que hemos inspeccionado arriba presuponen importantes implicaciones ontológicas y metodologicas de la doctrina laplaciana determinista que, como hemos hecho ver, son lógicamente independientes de la tesis determinista propiamente dicha. En particular se asume que las atribuciones de probabilidad se justifican sólo si reflejan ignorancia de ciertos hechos. En este caso los hechos que no conocemos corresponderían a propiedades 'finas' de los sistemas en cuestión, ciertas correlaciones entre moléculas por ejemplo. Se asume también que los sistemas físicos pueden y deben poder considerarse aislados de otros vecinos para llegar a formular una teoría adecuada sobre ellos. Si abandonamos estos preceptos metodológicos, sin embargo, podemos llegar a formular una noci ín de azar objetivo clara.

La mecánica estadística puede intepretarse no como una mecánica de colectivi 'ades en las que cada sistema es aislable, o más precisamente, separable (en el sentido de poder ser descrito idealmente, cuando menos, por completo con una descripción clásica de estado), sino como la teoría que describe el movimiento de sistemas mecánicos no aislables (en el sentido 
de tener estados no separables). ${ }^{9}$ Esto tiene como consecuencia que deba reconsiderarse la idealización usual en teorías de mecánica estadística que identifican el estado de un sistema físico con el estado de la mecánica clásica newtoniana. No tenemos por qué pensar que la imposibilidad de preparar un estado clásico es la única explicación posible para entender la presencia de un azar físico, como sería el caso si aceptamos los principios metodológicos laplacianos ya mencionados.

Si los sistemas se consideran constituidos por estados no separables, debemos pensar en los sistemas como "irreductiblemente" relativos a una situación física determinada. Un sistema en esta intepretación no es meramente un agregado de sus subsistemas. Éste es un tipo diferente de idealización. Diferentes teorías van a teorizar de forma diferente esta no separabilidad de los estados, pero lo que es importante para nosotros es que la introducción de estados no separables permite incorporar directamente en los fundamentos de una teoría estadística la imposibilidad física que genera el azar objetivo al que se refiere la irreductibilidad de las probabilidades.

Es más, podemos pensar que una teoría estadística de la mecánica es tal que, como sucede en la mecánica cuántica, los sistemas se caracterizan por dos tipos de estados diferentes, unos separables (estados estadísticos) y otros (estados individuales) no separables. Los estados separables están sujetos a una ley de evolución (más o menos) determinista que permite, por lo menos en ciertos casos, predecir los estados (estadísticos) futuros y la generación de distribuciones de azar aparente. Los estados no separables son los que nos permiten

9 Esta idea se encuentra desarrollada en Blatt (1959) como un intento de fundamentación filosófica del enfoque de Lebowitz et. al. Blatt, sin embargo, no establece la conexión, que nos parece crucial, entre esta interpretación de la mecánica estadística y la reformulación del concepto de estado mecánico. Blatt, además, está todavía tratando de fundamentar la idea de un azar objetivo en términos de los límites de nuestra capacidad de observación a partes finitas del universo. 
fundamentar un azar objetivo y en última instancia reconciliar la macro-descripción azarosa en teorías estadísticas con el movimiento supuestamente determinista de los sistemas a nivel microscópico. Este tema, sin embargo, tendrá que dejarse para un estudio posterior. ${ }^{10}$

\section{Conclusión}

Hay un candente debate en la filosofía de la ciencia entre los proponentes de diferentes interpretaciones de las probabilidades. Por un lado están los que sostienen que las probabilidades en la física son límites de frecuencias relativas. Por otro lado, están los que sostienen que las probabilidades son propensiones naturales cuantificadas. Creo que la teoría ergódica y otras teorías físicas modernas muestran de manera convincente, como se ha hecho ver arriba, la presencia de cierto tipo de propensiones en el mundo.

La discusión anterior refuerza nuestra creencia de que el problema filosófico de la interpretación de las probabilidades debe bajarse del cielo de la abstracción en el que lo han colocado los filosofos y dirigirse al examen cuidadoso de teorías específicas y del papel que juegan las probabilidades y otras modalidades físicas en estas teorías. Las discusiones filosóficas tradicionales parten de un análisis abstracto que solo parece generar controversia pero no un mayor entendimiento. Es posible que no existan propensiones en un sentido absoluto, pero que sí existan propensiones como importantes conceptos teóricos de teorías específicas. Esta idea de propensión estaría, por supuesto, asociada a una concepción no empirista de leyes de la naturaleza que no pretendo elucidar aquí.

10 Algunos aspectos de este tema, en particular la distinción entre dos tipos de estado en la mecánica cuántica, y el papel que juegan en la teoría, se examinan en Martínez 1990b. 


\section{REFERENCIAS BIBLIOGRÁFICAS}

Blatt, J. M. (1959), "An Alternative Approach to the Ergodic Problem", Prog. Theor. Physics, vol. 22, núm. 6, pp. 745-756.

Bennett (1985), "Dissipation, Information, Computational Complexity and Definition of Organization", en D. Pines (ed.), Emerging Syntheses in Science, Santa Fe Institute, Santa Fe.

Earman, J. (1986), A primer on Determinism, Reidel, Dordrecht.

Giere, R. (1973), "Objective-Single Case Probabilities and Foundations of Statistics", en Suppes, Henkin, Moisil, Joja (eds.), Logic, Methodology and Philosophy of Science, IV, North Holland, Amsterdam.

Hahn, E. (1953), “Free Nuclear Induction”, Physics Today, vol. 6, pp. 4-9.

Hopf, E. (1934), “On Causality, Statistics and Probability", Journal of Mathem. and Physics, vol. 17, pp. 51-102.

Krylov, N. (1979), Works on the Foundations of Statistical Mechanics, Princeton University Press, Princeton.

Laplace, P. S. (1814), Essay philosophique sur les Probabilités. Ensayo filosófico sobre las probabilidades, trad. española de P. Castrillo, Alianza, Madrid, 1985.

Martínez, S. (1990a), “El azar objetivo como medida matemática de desorden", de próxima aparición en Diánoia, 1990.

—_ (1990b), “Azar e indeterminismo en la mecánica cuántica", manuscrito.

Peres (1987), "Quantum Chaos and Irreversibility", Proccedings of the Symposium on the Foundations of Modern Physics: The Copenhagen Interpretation 60 years after the Como Lecture.

Plato, von J. (1982), "Probability and Determinism", J. Phil. of Sc., vol. 49 , pp. $51-66$.

(1988), "Ergodic Theory and the Foundations of Probability", en B. Skyrms and W. L. Harper (eds.), Causation, Chance and Credence, vol. I, pp. 257-277, Kluwer Academic Publishers.

Popper, K. (1962), "The Propensity Interpretation in the Calculus of Probability, and the Quantum Theory", en S. Körner (ed.), Observation and Interpretation in the Philosophy of Physics, Dover, Nueva York.

Prigogine, I. (1980), From Being to Becoming, W. H. Freeman, Nueva York. 
Salmon (1984), Scientific Explanation and the Causal Structure of the World, Princeton University Press, Princeton.

Skyrms, B. (1984), Pragmatics and Empiricism, Yale University Press, New Haven.

Solomonoff, R. J. (1964), “A Formal Theory of Inductive Inference. Part I", Information and Control, vol. 7, pp. 1-22.

Stegmüller, W. (1973), Probleme und Resultate der Wissenschaftstheorie und Analytischen Philosophie, tomo IV, Berlín, Springer Verlag.

Recibido: 21 mayo 1990. 


\section{SUMMARY}

This paper deals with the notion of objective randomness in classical deterministic theories. After the introduction, section 2 establishes an important distinction between a strictly metaphysical thesis of determinism (as characterized in the Montague-Earman definition, for example), and the doctrine of determinism, which can be roughly characterized as a methodological set of principles. The doctrine of determinism is associated with the idea that probability assignments can only reflect our ignorance of facts, and it also grounds the (ontological) thesis of separability: A system or process can be characterized completely in terms of the properties that a system has when in a given state, independently of the properties of other systems. The key notions of "completeness" and "independence" are only briefly disscussed, as they are examined more in depth elsewhere.

Section three examines attempts to characterize a notion of objective randomness in ergodic theory. The characterization can be seen to be equivalent to the formulation of a notion of a "physically impossible process". One way of expressing this idea is grounded on the thesis of the "coarse graining" of our measuring instruments. This leads to the problem of distinguishing "objective" from "apparent" randomness. The problem seems to be intrinsic to any attempt of characterizing the required notion of physical impossibility (and thus of objective randomness) in terms of an ideal observer. The alternative of trying to characterize physical impossibility in terms of a theory of algorithms suffers from the difficulty that it is not clear what would be the required notion of (non-epistemic) computability.

The "coarse graining" approach, as well as the alternative in terms of a theory of algorithms seem to share the usual confusion between a strictly metaphysical thesis of determinism and the (methodological) doctrine of determinism. In section four an alternative approach is suggested. It is noticed that a denial of the thesis of separability is compatible with a strict deterministic theory, and thus that at least some classical systems (the "statistical" ones) can be described in terms of non-separable states. We do not have to think that the impossibility of preparing a classical state is the only possible explanation for physical (objective) randomness in deterministic theories. This proposal is an elaboration of an idea of Blatt (1959), although 
Blatt was still trying to understand objective randomness within an ideal-observer framework.

[S. M.] 